\title{
Probing interstellar magnetic fields with Supernova remnants
}

\author{
Roland Kothes ${ }^{1}$ and Jo-Anne Brown ${ }^{2}$ \\ ${ }^{1}$ National Research Council of Canada, Herzberg Institute of Astrophysics, Dominion Radio \\ Astrophysical Observatory, P.O. Box 248, Penticton, British Columbia, V2A 6J9, Canada \\ email: Roland.Kothes@nrc-cnrc.gc.ca \\ ${ }^{2}$ Department of Physics and Astronomy, University of Calgary, 2500 University Drive N.W., \\ Calgary, AB, Canada \\ email: jocat@ras.ucalgary.ca
}

\begin{abstract}
As Supernova remnants expand, their shock waves are freezing in and compressing the magnetic field lines they encounter; consequently we can use Supernova remnants as magnifying glasses for their ambient magnetic fields. We will describe a simple model to determine emission, polarization, and rotation measure characteristics of adiabatically expanding Supernova remnants and how we can exploit this model to gain information about the large scale magnetic field in our Galaxy. We will give two examples: The SNR DA530, which is located high above the Galactic plane, reveals information about the magnetic field in the halo of our Galaxy. The SNR G182.4+4.3 is located close to the anti-centre of our Galaxy and reveals the most probable direction where the large-scale magnetic field is perpendicular to the line of sight. This may help to decide on the large-scale magnetic field configuration of our Galaxy. But more observations of SNRs are needed.
\end{abstract}

Keywords. Magnetic fields - polarization - ISM: individual (DA 530, G182.4+4.3) - ISM: magnetic fields - Supernova remnants

\section{Introduction}

Recently, there have been several studies of the Milky Way's large-scale magnetic field utilizing observations of the rotation measure of compact polarized objects like extra-galactic point sources (e.g. Brown et al. 2007) or pulsars (see A. Noutsos and J.L. Han, this proceedings). However, we still do not know the large-scale magnetic field configuration or even the number of field reversals within our Galaxy. One difficulty is that through Faraday rotation studies of compact extra-galactic sources we only derive the average magnetic field parallel to the line of sight $B_{\|}$through our Galaxy weighted by the electron density $n_{e}$. Here, the rotation measure $R M$ is given by

$$
R M=0.81 \int_{l} B_{\|} n_{e} d l .
$$

Here, $R M$ is given in $\mathrm{rad} / \mathrm{m}^{2}, B$ in $\mu \mathrm{G}, n_{e}$ in $\mathrm{cm}^{-3}$, and the pathlength $l$ in pc. In addition extragalactic sources may suffer from intrinsic Faraday rotation of unknown magnitude. Faraday rotation studies of pulsars average $B_{\|}$between us and the pulsar weighted by $n_{e}$. One significant problem of the averaging procedure is that there could be numerous field reversals along the line of sight, which would be averaged out. This ambiguity could be solved if we had anchor points for the magnetic field structure within our Galaxy. We propose to determine these anchor points with polarization and Faraday rotation studies of Supernova remnants (SNRs), since these can be used as magnifying glasses of their ambient magnetic field. 


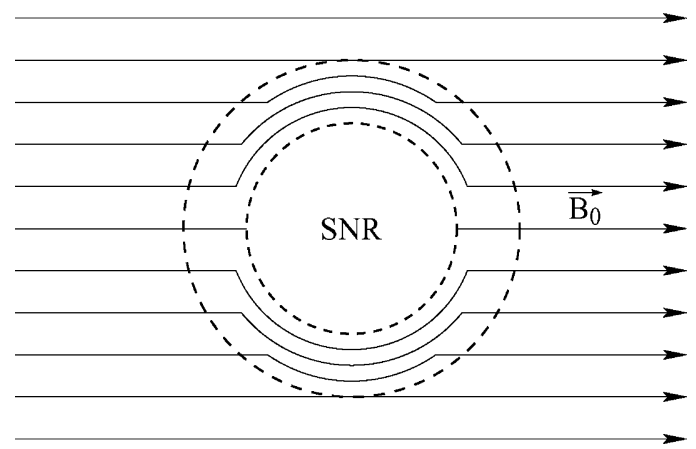

Figure 1. Simple model of the magnetic field inside an adiabatically expanding SNR.

\section{A simple model of Supernova remnants}

We assume a Supernova remnant, which is adiabatically expanding into an ambient medium of constant density $n_{0}$ and constant homogeneous magnetic field $\vec{B}_{0}$. In our model the SNR is spherical and has a shell with a width of about $10 \%$ of the SNR's radius in which the ambient medium and its magnetic field are compressed by a factor of 4. While the electron density in the shell is assumed to be constant the magnetic field changes with the angle between the SNR's expansion direction and the ambient magnetic field (see Fig. 1). It is proportional to the "number" of magnetic field lines, which are swept up by the expanding shock wave, hence the magnetic field inside the shell is strongest where the SNR is expanding perpendicular to the ambient magnetic field, and zero where it is parallel. The synchrotron emission is integrated along the line of sight from the back of the SNR to the front and appropriately Faraday rotated (see Equation 1.1). The synchrotron flux density $S$ produced by a spectrum of relativistic electrons $N(E)$ at frequency $\nu$ is given by

$$
S_{\nu} \propto K B_{\perp}^{\frac{1}{2}(\delta+1)} \nu^{-\frac{1}{2}(\delta+1)}, \quad N(E) d E=K E^{-\delta} d E .
$$

Here, $E$ is the energy and the values for $K$ and $\delta$ are defined by $N(E)$.

In Fig. 2 we display the simulated emission structure in total intensity and polarized intensity and the internal rotation measure that would be observed from a typical Supernova remnant at different viewing angles $\Theta$. In this simulation the ambient magnetic field is pointing away from us from the front left to the back right and $\Theta$ is the angle between the plane of the sky and the magnetic field lines the SNR is expanding into. For negative $\Theta$, the magnetic field would point towards us from the back left to the front right. In the simulation shown in Fig. 2 the ambient magnetic field is $4 \mu \mathrm{G}$, the ambient density is $1 \mathrm{~cm}^{-3}$, the distance to the SNR is $2 \mathrm{kpc}$, and its diameter is $20 \mathrm{pc}$. The simulation was convolved with a beam of 2.5 '.

In emission we find the typical bilateral structure of SNRs in total power and polarized intensity with a tangential magnetic field up to a $\Theta$ of about $60^{\circ}$, beyond which the SNR becomes circular and thick-shelled with a radial magnetic field. The surface brightness of the SNR is decreasing from $\Theta=0^{\circ}$ to $\Theta=90^{\circ}$ by a factor of almost 10 . The RM structure reveals a few very interesting characteristics. The internal rotation measure in the centre of the SNR is always zero, which indicates that from observations we can determine the foreground $R M$ there. The entire SNR except for $\Theta=0^{\circ}$ is dominated by RM of one sign; in Fig. 2 it is mostly negative. This indicates whether the ambient magnetic field is pointing away from us or towards us. There is also a smaller area of opposite sign. Its location indicates the orientation of the ambient magnetic field and its 

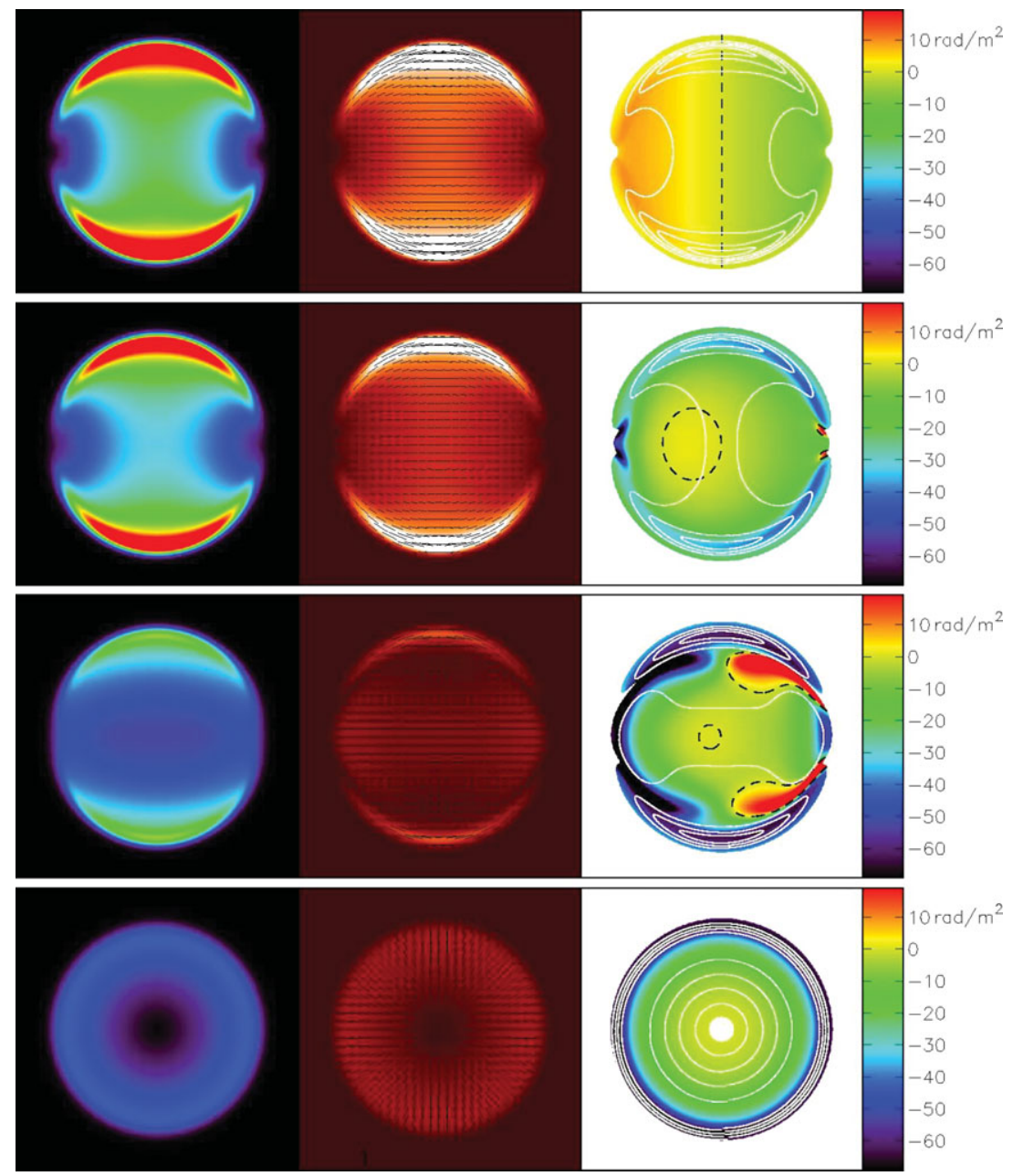

Figure 2. Simulated emission structure in Stokes I (left column), polarized intensity (centre column) with overlaid B-vectors, and RM (right column) with overlaid white contours indicating the polarized emission for different angles $\Theta$ (from top to bottom: $\Theta=0^{\circ}, 30^{\circ}, 60^{\circ}$, and $90^{\circ}$ ).
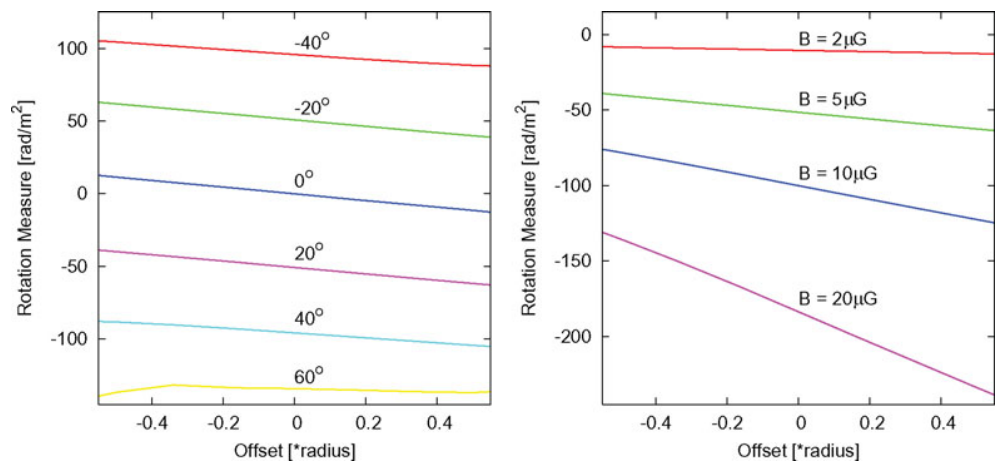

Figure 3. The rotation measure gradient on the arcs of the SNR as a function of the distance from their centres in the simulation described in section 2.2. Left: $\Theta$ varies from $-40^{\circ}$ (top) to $+60^{\circ}$ (bottom). Right: $\Theta=20^{\circ}$ and $\left|\overrightarrow{B_{0}}\right|$ varies from $2 \mu \mathrm{G}$ (top) to $20 \mu \mathrm{G}$ (bottom). 

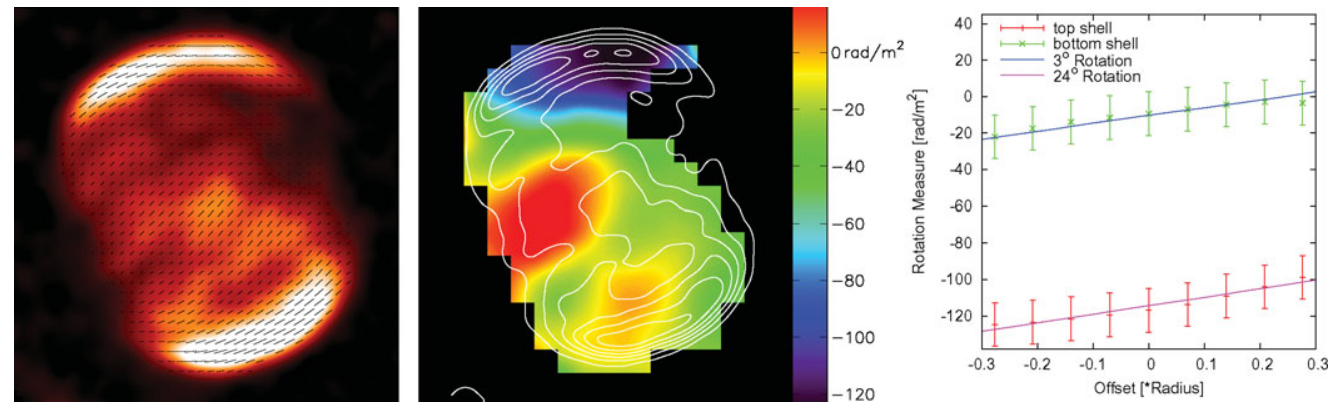

Figure 4. Left: Polarized intensity map of the SNR DA 530 at $4.85 \mathrm{GHz}$, observed with the $100 \mathrm{~m}$ telescope in Effelsberg. Vectors in B-field direction are overlaid. Centre: Rotation measure map calculated between $4.85 \mathrm{GHz}$ and $10.45 \mathrm{GHz}$ (100 m Effelsberg) of SNR DA 530 with overlaid white contours indicating polarized intensity. Right: Observed rotation measure gradients on the shells of the SNR DA 530 as a function of distance from the centre of the shell.

distance from the centre gives an approximate value for $\Theta$. In addition we find that the $\mathrm{RM}$ on the two arcs shows a linear behaviour up to about $\Theta=60^{\circ}$ (see Fig. 3). This can be described by its gradient, which depends entirely on $\left|\overrightarrow{B_{0}}\right| \times n_{0}$ and the size of the SNR and is almost independent of $\Theta$ and the $R M$ in the middle, which is determined from $\Theta$ and the foreground $R M$. To show how we can exploit these characteristics we will give two examples.

\section{The SNR DA 530 and the Galactic halo}

In Fig. 4 we display a polarized intensity and rotation measure map of the SNR DA 530, which is located high above the Galactic plane at a latitude of about $+7^{\circ}$. The overlaid vectors in the polarized intensity image indicate a tangential magnetic field, which we expected from the simulations for a SNR with a $\Theta$ of less than $60^{\circ}$. The rotation measure in the centre is about $0 \mathrm{rad} / \mathrm{m}^{2}$, hence we can neglect foreground effects. The RM map is dominated by negative rotation measures, but there is a small area of positive RM to the left of the centre. This indicates that the SNR is expanding into a magnetic field, which is pointing away from the front left to the back right.

As can be seen in Fig. 4 the RM values on the arcs differ significantly, however, the gradient seems to be the same. This implies that $n_{0}$ and $\left|\overrightarrow{B_{0}}\right|$ are the same for both shells. The difference could be either in the foreground RM or $\overrightarrow{B_{0}}$ for the two arcs have a different $\Theta$. To find such a large difference in RM on such a small scale in the foreground is very unlikely, since it would require either another SNR or an HII region along the line of sight to produce such a large effect, both of which would be easily detectable by other means. The only possibility left is a twisted ambient magnetic field. Simulations for both shells indicate that the top shell is expanding into a magnetic field with $\Theta=24^{\circ}$ and the bottom one with $\Theta=3^{\circ}$. The lower surface brightness of the top shell supports this finding. The radio surface brightness goes down with $\Theta$, because the magnetic field inside the SNR is more and more along the line of sight (see Equation 2.1).

Radio observations of other galaxies show twisted magnetic spurs emerging from star forming regions (e.g. Review by Beck 2008). DA 530 is located above an area of the Milky Way, which is rich in star forming regions, HII regions, and SNRs. Is DA 530 expanding inside these twisted magnetic spurs? 

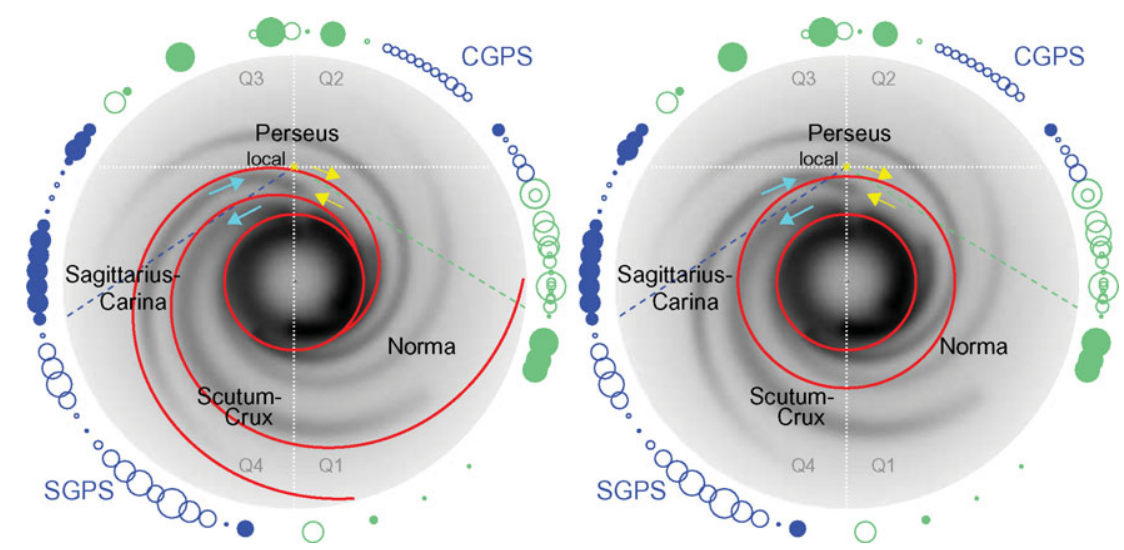

Figure 5. Extragalactic rotation measures and the large-scale magnetic field of the Milky Way - a birds-eye-view. Filled symbols indicate positive RM, open circles indicate negative RM, and the size of the circles are proportional to the RM. The gray-scale is the electron density model of Cordes \& Lazio (2002). The arrows in the first quadrant (Q1) indicate the magnetic field directions commonly accepted, while the arrows in the fourth quadrant (Q4) indicate the magnetic field directions as determined from Brown et al. (2007). The solid lines are suggested spiral field lines (left) and circular field lines (right). The common names of the spiral arms are also labeled.

\section{The SNR G182.4+4.3 and the Galactic anti-centre}

In terms of the large-scale Galactic magnetic field, it is generally accepted that the field is directed clockwise in our local arm, as viewed from the North Galactic pole, and that there is at least one large-scale magnetic field reversal (a region of magnetic shear where the field is seen to reverse directions by roughly $180^{\circ}$ ) in the inner Galaxy between the local and Sagittarius-Carina arms (Simard-Normandin \& Kronberg 1979; Thomson \& Nelson 1980), as indicated by the filled arrows in quadrant 1 (Q1) in Fig. 5. Further to the existence and location of additional magnetic field reversals is the question of field alignment with the optical spiral arms. It is often assumed that the field is closely aligned with the spiral arms. Brown et al. (2007) demonstrated conclusively that the field in the fourth quadrant (Q4) of the Sagittarius-Carina arm is directed clockwise, the same as the local field. This presents a continuity problem if the field does indeed follow the optical arms, since the field is known to be counter-clockwise in Q1 of the Sagittarius-Carina arm, as shown in Fig. 5 (left). However, if the field is much less inclined than the optical spiral arms, perhaps even purely azimuthal as suggested by Vallée (2005), the continuity problem is resolved, as shown in Fig. 5 (right). Additional information regarding the field alignment may be found towards the anti-centre region of the Milky Way. If the largescale magnetic field of our Galaxy is azimuthal the magnetic field component parallel to the line of sight $B_{\|}$would be 0 towards a Galactic longitude of $180^{\circ}$; if the magnetic field follows the spiral arms we would expect $B_{\|}=0$ towards Galactic longitude between $165^{\circ}$ and $170^{\circ}$.

The SNR G182.4+4.3 (Fig. 6) is conveniently located close to the anti-centre at a Galactic longitude of $182.4^{\circ}$. Unfortunately only one shell of this SNR is visible, likely because the bottom shell is expanding towards the Galactic plane into higher density and the top shell away from the plane into very low density. Without emission from the centre of the SNR we cannot easily determine the foreground $R M$. On the bottom shell we find a RM gradient which goes from about 0 in the right to about $50 \mathrm{rad} / \mathrm{m}^{2}$ on the left (Fig. 6). This implies that the ambient magnetic field is pointing towards us from back left to front right. This is not surprising, since the magnetic field in the outer 

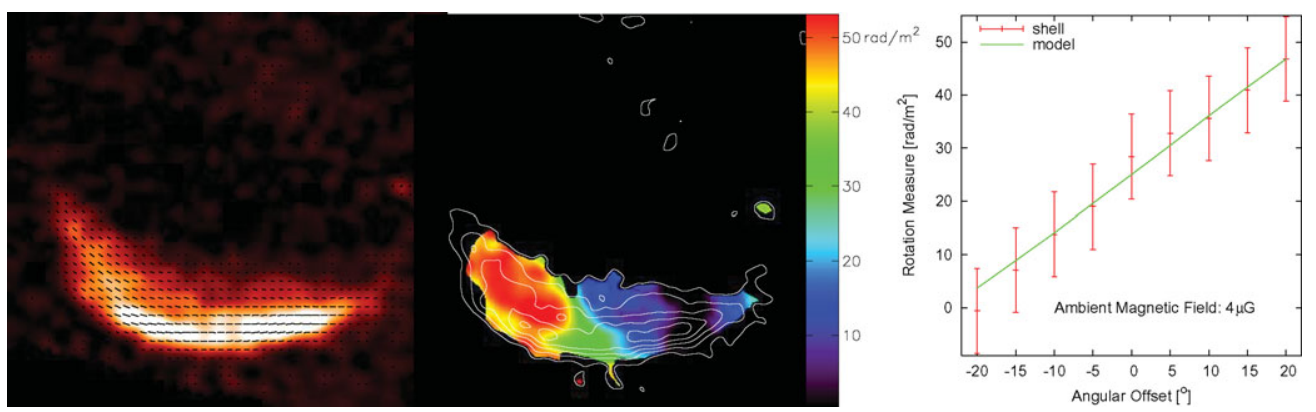

Figure 6. The same as Fig. 4 but for the SNR G182.4+4.3. The rotation measure map was calculated between $4.85 \mathrm{GHz}$ (100 m Effelsberg) and $1420 \mathrm{MHz}$ taken from the Canadian Galactic Plane Survey (CGPS, Taylor et al. 2003).

Galaxy should be directed from higher to lower longitudes and we are here at a longitude which is higher than the $B_{\|}=0$ point for both possible magnetic field configurations. Neglecting the foreground contribution for the RM we can determine an upper limit for $\Theta$ by simulating a SNR for the RM map of G182.4+4.3. The result is independent of the distance to G182.4+4.3. We derive $\Theta \geqslant-4^{\circ}$, hence the Galactic longitude at which $B_{\|}=0$ must be larger than $178^{\circ}$. If we now assume that the foreground magnetic field has the same $\Theta$ as the SNR's ambient field and the foreground field is constant at $4 \mu \mathrm{G}$, which would be a reasonable assumption, we would find $\Theta=-2^{\circ}$. This supports the assumption that the Milky Way has an azimuthal magnetic field structure.

\section{Acknowledgements}

The Dominion Radio Astrophysical Observatory is a National Facility operated by the National Research Council Canada. The Canadian Galactic Plane Survey is a Canadian project with international partners, and is supported by the Natural Sciences and Engineering Research Council (NSERC). This research is based on observations with the 100-m telescope of the MPIfR at Effelsberg.

\section{References}

Beck, R. 2008, astro-ph 0711.4700

Brown, J. C., Haverkorn, M., Gaensler, B. M., Taylor, A. R., Bizunok, N. S., McClure-Griffiths, N. M., Dickey, J. M., \& Green, A. J. 2007, ApJ 663, 258

Cordes, J. M. \& Lazio, T. J. W. 2002, astro-ph 0207156

Simard-Normandin, M. \& Kronberg, P. P. 1979, Nature 279, 115

Taylor, A. R., Gibson, S. J., Peracaula, M., et al. 2003, AJ 124, 3145

Thomson, R. C. \& Nelson, A. H. 1980, MNRAS 191, 863

Vallée, J. P. 2005, ApJ 619, 297

\section{Discussion}

GAEnsler: Does your technique only work for old SNRs in which synchrotron emission and polarizations comes from swept-up gas? Most observable SNRs are younger and have emissions coming from turbulent reverse shock.

Kothes: It should work for all Supernova remnants, which show the typical shell structure with a tangential magnetic field. It will not work for very young SNRs expanding in complex media. 